\title{
Visual Representations by Cortical Somatostatin Inhibitory Neurons-Selective But with Weak and Delayed Responses
}

\author{
Wen-pei Ma, ${ }^{1 \star}$ Bao-hua Liu, ${ }^{1,4 \star}$ Ya-tang Li, ${ }^{1,4}$ Z. Josh Huang, ${ }^{5}$ Li I. Zhang, ${ }^{1,3}$ and Huizhong W. Tao ${ }^{1,2}$ \\ 1Zilkha Neurogenetic Institute, Departments of ${ }^{2}$ Cell and Neurobiology and ${ }^{3}$ Biophysics and Physiology, and ${ }^{4}$ Graduate Programs, University of Southern \\ California, Los Angeles, California 90089, and ${ }^{5}$ Cold Spring Harbor Laboratory, Cold Spring Harbor, New York 11724
}

\begin{abstract}
Somatostatin-expressing inhibitory (SOM) neurons in the sensory cortex consist mostly of Martinotti cells, which project ascending axons to layer 1. Due to their sparse distribution, the representational properties of these neurons remain largely unknown. By twophoton imaging guided cell-attached recordings, we characterized visual response and receptive field (RF) properties of SOM neurons and parvalbumin-expressing inhibitory $(\mathrm{PV})$ neurons genetically labeled in the mouse primary visual cortex. In contrast to PV neurons, SOM neurons exhibit broader spikes, lower spontaneous firing rates, smaller $\mathrm{On} / \mathrm{Off}$ subfields, and broader ranges of basic RF properties such as $\mathrm{On} / \mathrm{Off}$ segregation, orientation and direction tunings. Notably, the level of orientation and direction selectivity is comparable to that of excitatory neurons, from weakly-tuned to highly selective, whereas PV neurons are in general unselective. Strikingly, the evoked spiking responses of SOM cells are $\sim 3$ - to 5-fold weaker and 20-25 ms delayed compared with those of PV neurons. The onset latency of the latter is consistent with that of inhibitory input to excitatory neurons. These functional differences between SOM and PV neurons exist in both layer 2/3 and 4. Our results suggest that SOM and PV neurons engage in cortical circuits in different manners: while PV neurons provide fast, strong but untuned feedforward inhibition to excitatory neurons, likely serving as a general gain control for the processing of ascending inputs, SOM neurons with their selective but delayed and weak inhibition may provide more specific gating of later arriving intracortical excitatory inputs on the distal dendrites.
\end{abstract}

\section{Introduction}

Cortical GABAergic inhibitory neurons are morphologically, electrophysiologically and chemically diverse (Kawaguchi and Kubota, 1997; Markram et al., 2004). The respective contribution of each subtype of inhibitory neurons to cortical processing remains largely undetermined. The major difficulty exists in how to specifically target these sparsely distributed cells in vivo. The newly developed two-photon imaging guided recording (Margrie et al., 2003; Liu et al., 2009; Gentet et al., 2010) of genetically labeled specific types of neurons provides a promising approach for systematically addressing this issue. Despite the complexity of their morphological or electrophysiological features, inhibitory neurons are known to contain major neurochemically defined groups (Gonchar and Burkhalter, 1997; Kawaguchi and Kubota, 1997). For example, in the mouse visual cortex, parvalbumin $(\mathrm{PV})$, calretinin (CR) and somatostatin (SOM) are expressed in $39 \%, 24 \%$ and $23 \%$ of GABAergic neurons respectively (Gonchar et al., 2007). PV-positive neurons are distinct from CR- and SOM-positive neurons, while there is a partial overlap between CR- and SOM-expressing cells (Gonchar et al., 2007; Xu et al.,

Received June 23, 2010; revised Aug. 25, 2010; accepted Sept. 6, 2010.

This work was supported by grants from the National Institutes of Health (NIH) (EY018718 and EY019049) and The Kirchgessner Foundation to H.W.T. L.I.Z. received support from the NIH-National Institute on Deafness and Other Communication Disorders, the Searle Scholar Program, the Klingenstein Foundation, and the David and Lucile Packard Foundation.

*W.-p.M. and B.-h.L. contributed equally to the work.

Correspondence should be addressed to: H. W. Tao at the above address. E-mail: htao@usc.edu.

DOI:10.1523/JNEUROSCI.3248-10.2010

Copyright $\odot 2010$ the authors $\quad$ 0270-6474/10/3014371-09\$15.00/0
2010), unlike in the rat cortex (Gonchar and Burkhalter, 1997). Thus, the three chemically specified subtypes together can account for a majority of total inhibitory neurons.

Previous studies have shown that PV and SOM neurons preferentially target perisomatic and more distal dendritic domains of principal neurons, respectively (Freund and Buzsáki, 1996; Somogyi et al., 1998; Di Cristo et al., 2004). Based on these differential subcellular targeting preferences, it has been postulated that $\mathrm{PV}$ and SOM cells play distinct roles in regulating principal neurons' responses. However, the processing properties of SOM neurons and their functional distinctions compared with PV neurons remain unclear. In an earlier study (Liu et al., 2009), using two-photon imaging guided cell-attached recordings in GAD67-GFP knock-in transgenic mice, we examined the receptive field (RF) properties of inhibitory neurons in layer $2 / 3$ of the primary visual cortex (V1). The recorded neurons were categorized based on spike shape (fast-spike, FS, and regular-spike, RS), and relatively uniform RF properties were found for FS and RS inhibitory neurons (Liu et al., 2009). Although the FS neurons were most likely PV cells (Kawaguchi and Kubota, 1997), the RS inhibitory neurons were a mixture of other GABAergic subtypes. In the present study, we applied the targeted recording technique to more specific transgenic lines in which SOM and PV neurons are genetically labeled respectively, and extended our investigations to layer 4. Interestingly we found that SOM neurons exhibit distinct functional properties from PV neurons. In contrast to generally nonselective PV neurons, SOM neurons display robust selectivity for orientation and direction, resembling excitatory neurons. More strikingly, visual responses of SOM neurons are 
markedly weaker and delayed compared with PV neurons. Together with the morphological features of these two types of inhibitory neurons, we propose that while PV neurons provide a fast and strong general gain control, SOM neurons may specifically modulate later arriving intracortical excitatory inputs on the distal dendrites of principal neurons.

\section{Materials and Methods}

Animal preparation. All experimental procedures were approved by the Institutional Animal Care and Use Committee of University of Southern California. The G42 mouse line was maintained by crossing heterozygous G42 with CB6F1/J. The GIN mice were obtained by breeding homozygous GIN with C57BL/6. Heterozygous offspring were used for imagingguided recording experiments. Wild-type C57BL/6 mice were used for blind recording experiments. Adult female mice ( $>90 \mathrm{~d}$ old) were anesthetized with urethane $(1.2 \mathrm{~g} / \mathrm{kg})$ and sedative chlorprothixene $(0.05 \mathrm{ml}$ of $4 \mathrm{mg} / \mathrm{ml}$ ). The surgery was performed as previously described (Niell and Stryker, 2008; Liu et al., 2009). The exposed cortical surface was applied with artificial CSF (ACSF) (containing in mM: $140 \mathrm{NaCl}, 2.5 \mathrm{KCl}$, $2.5 \mathrm{CaCl}_{2}, 1.3 \mathrm{MgSO}_{4}, 1.0 \mathrm{NaH}_{2} \mathrm{PO}_{4}, 20$ HEPES, 11 glucose, $\mathrm{pH} 7.4$ ) when necessary.

In vivo two-photon imaging-guided recording and blind loose-patch recording. In vivo two-photon imaging was performed with a custombuilt imaging system. A mode-locked Ti:sapphire laser (MaiTai Broadband, Spectra-Physics) was tuned at $890 \mathrm{~nm}$ with the output power at $10-60 \mathrm{~mW}$ for $\mathrm{L} 2 / 3$ neurons and $80-200 \mathrm{~mW}$ for L4 neurons, adjusted according to the cell's fluorescence level. Note that fluorescence in G42 line is weaker than in GIN line. For cell-attached recording, the glass electrode, with $\sim 1 \mu \mathrm{m}$ tip opening and 8-10 $\mathrm{M} \Omega$ impedance, was filled with ACSF containing $0.15 \mathrm{~mm}$ calcein (Invitrogen). The pipette capacitance was completely compensated. The pipette tip was navigated in the cortex and patched onto a fluorescent soma as previously described (Liu et al., 2009). After confirming a successful targeting (Liu et al., 2009), the positive pressure in the pipette $(\sim 10 \mathrm{mbar})$ was then released and a negative pressure (20-150 mbar) was applied to form a loose seal (with $80-200 \mathrm{M} \Omega$ resistance), which was maintained throughout the course of the recording. Spike responses were recorded with an Axopatch 200B amplifier (Molecular Devices). Loose-patch recording was made under voltage-clamp mode and a command potential was adjusted so that the baseline current was $0 \mathrm{pA}$. The recorded signal was filtered at $10 \mathrm{kHz}$ and sampled at $50 \mathrm{kHz}$. The depth of the patched cell was directly determined under imaging. For blind recordings, glass electrodes with relatively larger tip openings (5-7 $\mathrm{M} \Omega$ impedance) were used. With this parameter, all the neurons blindly recorded showed regular spikes, suggesting that sampling was highly biased toward excitatory neurons. Neurons assigned to layer $2 / 3$ were located $100-350 \mu \mathrm{m}$ beneath the pia, and neurons assigned to layer 4 were at a depth of $375-500 \mu \mathrm{m}$.

Visual stimulation. Software for data acquisition and visual stimulation was custom-developed using LabView (National Instruments) and MATLAB (MathWorks). Visual stimuli were provided by a $34.5 \times 25.9$ $\mathrm{cm}$ monitor (refresh rate $120 \mathrm{~Hz}$, mean luminance $\sim 12 \mathrm{~cd} / \mathrm{m}^{2}$ ) placed 25 $\mathrm{cm}$ away from the right eye. The center of the monitor was placed at $45^{\circ}$ azimuth (corresponding to the monocular zone), $0^{\circ}$ elevation, and it covered $\pm 35^{\circ}$ horizontally and $\pm 27^{\circ}$ vertically of the visual field of the mouse. To map spatial RFs, bright and dark squares over a gray background (contrast $70 \%$ and $-70 \%$ respectively) within an $11 \times 11$ grid $\left(\right.$ grid size $5^{\circ}$ ) were flashed individually (duration $=200 \mathrm{~ms}$, interstimulus interval $=300 \mathrm{~ms}$ ) in a pseudo-random sequence. The sign of contrast (On or Off) was determined randomly. Each location was stimulated for 8-24 times, and the same number of On and Off stimuli were applied. One-dimensional RF was also mapped with 15 flash bright/dark bars $\left(3^{\circ}\right)$ of preferred orientation. The On and Off subfields were derived from responses to the onset of bright and dark stimuli respectively. To measure orientation tuning, drifting sinusoidal gratings of 12 directions ( $30^{\circ}$ step) with temporal frequency of $2 \mathrm{~Hz}$ and spatial frequency $0.04 \mathrm{cycle} /{ }^{\circ}$ were presented on the full screen for $2 \mathrm{~s}$ with an interstimulus interval of $5.5 \mathrm{~s}$. The grating started to drift $5 \mathrm{~s}$ after it appeared on the screen, and stopped drifting for $0.5 \mathrm{~s}$. Grating of another orientation then appeared immedi- ately. The mean luminance of the screen was thus kept constant. The 12 patterns were presented in a random sequence, and were repeated 5-10 times. For the measurement of response modulation, drifting sinusoidal gratings of preferred direction (with temporal frequency of $2 \mathrm{~Hz}$ ) were presented for $50-100$ cycles, at various spatial frequencies $(0.01,0.02$, $0.04,0.08,0.16,0.32 \mathrm{cycle}^{\circ}$ ).

Data analysis. To analyze the spike shape, 50 individual spike waveforms (without filter application) were averaged. For flashing stimuli, stimulus-evoked spikes were counted within a $150 \mathrm{~ms}$ time window starting at the response onset. To quantify the evoked firing rate for each cell, responses to $4-5$ flash stimuli at the RF center were selected to calculate average firing rate (baseline subtracted). For drifting gratings, spikes were counted within a 70-2000 ms window after the onset of the drift. The baseline activity (average spike number in the same length of duration before the onset of stimuli) was subtracted from stimulus-evoked spike numbers. To analyze RF structure, subfield was identified as an area where pixels with significant evoked responses (with peak firing rate larger than 3 SDs of baseline level) were spatially contiguous. On and Off subfields were fitted with 2-D Gaussian ellipses. The outline of the ellipse was determined as such that it could cross as many pixels at the boundary as possible. An overlap index (OI) (Hirsch et al., 2003) was calculated for cells exhibiting both On and Off subfields. The OI is defined as:

$$
\mathrm{OI}=\frac{0.5 W_{1}+0.5 W_{2}-d}{0.5 W_{1}+0.5 W_{2}+d}
$$

where $d$ is the distance between the centers of two ellipses, $W_{1}$ and $W_{2}$ are the widths of them respectively, which are the segments of the line that connects the two centers intercepted by the ellipses.

The modulation ratio $M=R\left(F_{1}\right) / R\left(F_{0}\right)$ was calculated for responses to gratings at optimal spatial frequency. The poststimulus spike time histogram (PSTH) was first generated from all the cycles. $R\left(F_{1}\right)$ was calculated from the PSTH as the amplitude of the best-fitting sinusoid at the modulation frequency (Mata and Ringach, 2005). $R\left(F_{0}\right)$ was the mean spike rate during the drifting grating stimulus (baseline subtracted).

The strength of orientation selectivity was quantified with a global measure of orientation selectivity (Dragoi et al., 2000):

\section{Global OSI =}

$$
\sqrt{\left(\sum_{i}\left(R\left(\theta_{i}\right) * \sin \left(2 \theta_{i}\right)\right)\right)^{2}+\left(\sum_{i}\left(R\left(\theta_{i}\right) * \cos \left(2 \theta_{i}\right)\right)\right)^{2}} / \sum_{i} R\left(\theta_{i}\right) .
$$

$\theta_{i}$ is the angle of the moving direction of the grating. $R\left(\theta_{i}\right)$ is the spike response amplitude (with baseline subtracted) at angle $\theta_{i}$. The direction selectivity index (DSI) was defined as $\left(R_{\text {pref }}-R_{\text {null }}\right) /\left(R_{\text {pref }}+R_{\text {null }}\right)$, $R_{\text {pref }}$ is the maximum response and $R_{\text {null }}$ is the response at the opposite direction.

To quantify the onset latency of evoked responses, PSTH was generated from spikes evoked by all the flash stimuli (bin size $=4 \mathrm{~ms}$ ). The onset of spiking responses was defined as the time point at which firing rate exceeded 3 SDs of baseline level. For statistical analysis, Tamhane T2 multiple-comparison test was performed for comparisons among multiple groups, unless otherwise indicated.

\section{Results}

Targeted cell-attached recording from SOM and PV neurons We took advantage of available transgenic lines in which SOM and PV neurons are genetically labeled, respectively (Oliva et al., 2000; Chattopadhyaya et al., 2004). In GIN (GFP-expressing inhibitory neurons) line, green fluorescence protein (GFP) is almost exclusively expressed by SOM neurons in the V1, and $72 \%$ and $57 \%$ of SOM neurons are labeled by GFP in layer $2 / 3$ and 4 , respectively (Xu et al., 2006). Fluorescent neurons are mainly distributed in layers $2 / 3$, layer 4 and upper layer 5 of the cortex (Fig. 1 A), as previously reported (Oliva et al., 2000). The majority of these fluorescent neurons exhibit bitufted morphology with vertically or obliquely orientated primary dendrites, and they all 
A

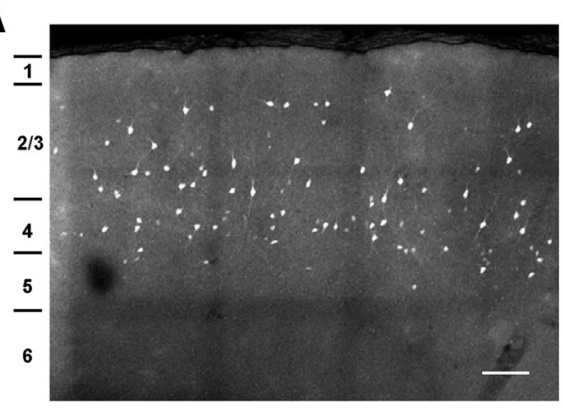

D

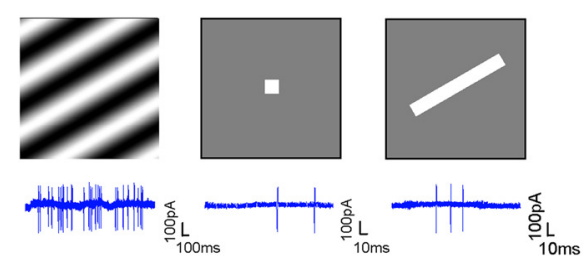

B

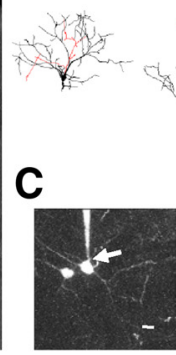

E

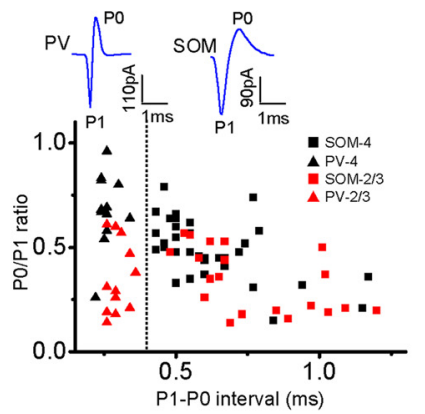

$\mathbf{F}$

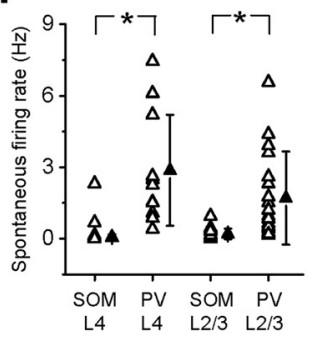

G

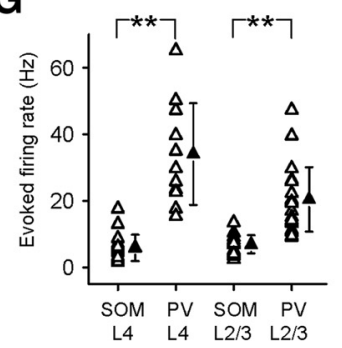

H

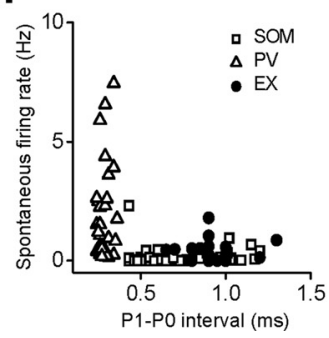

Figure 1. Spiking properties of SOM and PV neurons. $A$, Confocal image of a coronal section of the V1 of a GIN mouse. Cortical layers are indicated. Scale bar, $120 \mu \mathrm{m}$. $\boldsymbol{B}$, Traced morphology of example SOM neurons with their laminar locations indicated (right). Dendrites and soma are labeled in black, and axons in red. Scale bar, $120 \mu \mathrm{m}$. C, Two-photon image of a targeted SOM neuron at a depth of $400 \mu \mathrm{m}$ below the pia. The arrow points to the tip of the recording pipette, which was filled with ACSF containing calcein. Scale bar, $20 \mu \mathrm{m}$. D. Top, Example images of three different types of visual stimulation: drifting sinusoidal gratings (left), flashing squares (middle) and flashing bars (right). Bottom, Example evoked spike responses under the corresponding stimulation. $\boldsymbol{E}$, Scatter plot of P0/P1 ratio versus P1-P0 interval for the recorded SOM and PV neurons. P1 and P0 represent the negative and positive peak of the average spike shape, respectively. Top, Average spike shape for an example PV (left) and SOM (right) neuron. F, Spontaneous firing rate for SOM and PV neurons in layer 4 (L4) and 2/3 (L2/3). Solid symbol indicates the average value within the group. Error bar, SD. $N=37,12,25$, and 21 (from left to right). ${ }^{*} p<0.05$. G, Average evoked firing rate. $N=20$, 12,18 , and 20. ${ }^{* *} p<0.01$. $\boldsymbol{H}$, Scatter plot of spontaneous firing rate versus P1-P0 interval for the SOM, PV, and excitatory (Ex) neurons.

exhibited an ascending axon extending into layer 1, where the axon ramified (Fig. 1B) (see also Xu et al., 2006). These morphological features are characteristic of Martinotti cells (Fairén et al., 1984; Kawaguchi and Kubota, 1996; Wang et al., 2004). In G42 mice, GFP is only expressed in PV neurons, and $\sim 50 \%$ of PV cells are labeled (Chattopadhyaya et al., 2004; Xu and Callaway, 2009). Fluorescent neurons span all cortical layers except layer 1 , and they have been physiologically described predominantly as FS basket cells (Chattopadhyaya et al., 2004; Sugino et al., 2006; Xu and Callaway, 2009). In the V1 of GIN and G42 mice, we performed two-photon imagingguided patch-clamp recordings (TPTP) to target GFP-labeled SOM and PV neurons (in layer 2-4), respectively (Fig. 1C). Cell-attached recordings allowed us to detect spike signals from the patched neurons without affecting their intracellular milieu. Excitatory neurons were also blindly recorded in wild-type mice and identified by the regular-spike shape (see Materials and Methods).

We recorded the neurons' spike responses to three types of visual stimulation: drifting sinusoidal gratings, flashing bright/dark

squares as well as flashing bright/dark bars of optimal orientation (Fig. 1D) (see Materials and Methods). SOM and PV neurons exhibited distinct spike waveforms (Fig. $1 E)$. PV neurons had spikes with short trough-to-peak (P1-P0) intervals (in layer $2 / 3,0.30 \pm 0.03 \mathrm{~ms}$; in layer $4,0.27 \pm 0.05$ ms, mean $\pm S D)$, consistent with the notion that these neurons are FS cells (Kawaguchi and Kubota, 1997; Sugino et al., 2006; Xu and Callaway, 2009). SOM neurons exhibited significantly longer P1-P0 intervals than PV neurons $(p<0.01, t$ test; in layer $2 / 3,0.77 \pm 0.21 \mathrm{~ms}$; in layer $4,0.63 \pm 0.20$ $\mathrm{ms}$, mean $\pm \mathrm{SD})$. These two types of neurons can be separated by a P1-P0 interval of $0.4 \mathrm{~ms}$ (Fig. 1E). Thus, SOM neurons mostly belong to the regular-spike (RS) inhibitory neuron group according to the previous categorization (Liu et al., 2009). Interestingly, SOM neurons displayed a broad range of $\mathrm{P} 1-\mathrm{P} 0$ intervals, reminiscent of a previous report in the mouse cortex showing that SOM neurons that do not express calretinin have narrower action potential widths than those expressing calretinin (Xu et al., 2006). However, we did not find a significant bimodal distribution of $\mathrm{P} 1-\mathrm{P} 0$ intervals for the population of SOM cells ( $p>0.05$, Hartigan's dip test). On average, SOM neurons had significantly lower spontaneous firing rates than PV neurons (Fig. $1 F)$. They also responded to visual stimuli at significantly lower firing rates than PV neurons (Fig. 1G). Thus, the two subtypes of inhibitory neurons differ in their spiking activity profiles. On the other hand, SOM neurons resemble excitatory neurons in the level of spontaneous firing and spike shape (Fig. 1H).

\section{Receptive field properties of SOM and PV neurons in layer 4}

Layer 4 (L4) is the major thalamorecipient layer in the visual cortex (Felleman and Van Essen, 1991; Callaway, 1998). Analysis of L4 responses is crucial for the understanding of the first stage of cortical processing. We first examined the RF properties of L4 inhibitory neurons. An example SOM neuron is shown in Figure $2 \mathrm{~A}$. Under flashing squares, the neuron exhibited clearly identifiable On and Off spiking response regions (i.e., On and Off subfields), which overlapped considerably with each other (Fig. $2 A_{1}$ ). Mapping with bars of optimal orientation also revealed largely overlapping On and Off subfields (Fig. $2 A_{1}$, bottom right). The neuron responded to the drifting sinusoidal grating at optimal orientation and spatial frequency with weak modulation, as indicated by the low value of modulation ratio, which is the ratio of the first harmonic of the response at the temporal frequency of stimulus (F1 component) over the mean firing rate (F0) (Fig. $2 A_{2}$ ). Such weak modulation of responses (i.e., F1/F0 ratio $<1$ ) correlates well with the RF structure that has overlapping On and Off subfields (Liu et al., 2009). When tested with gratings moving at various directions, the SOM cell displayed a clear preference to vertical 

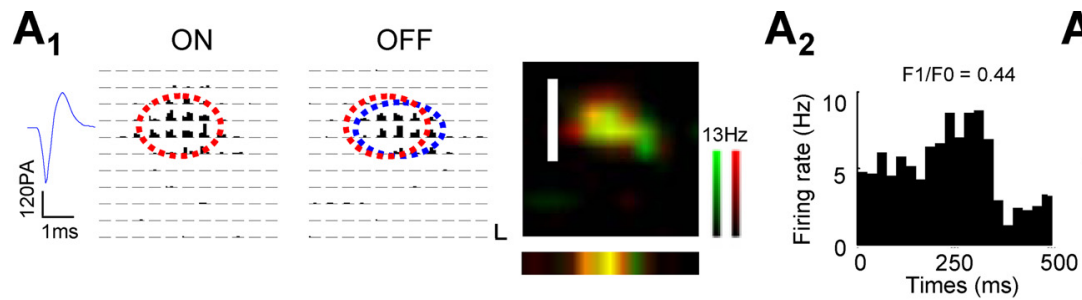

$\mathbf{A}_{3}$
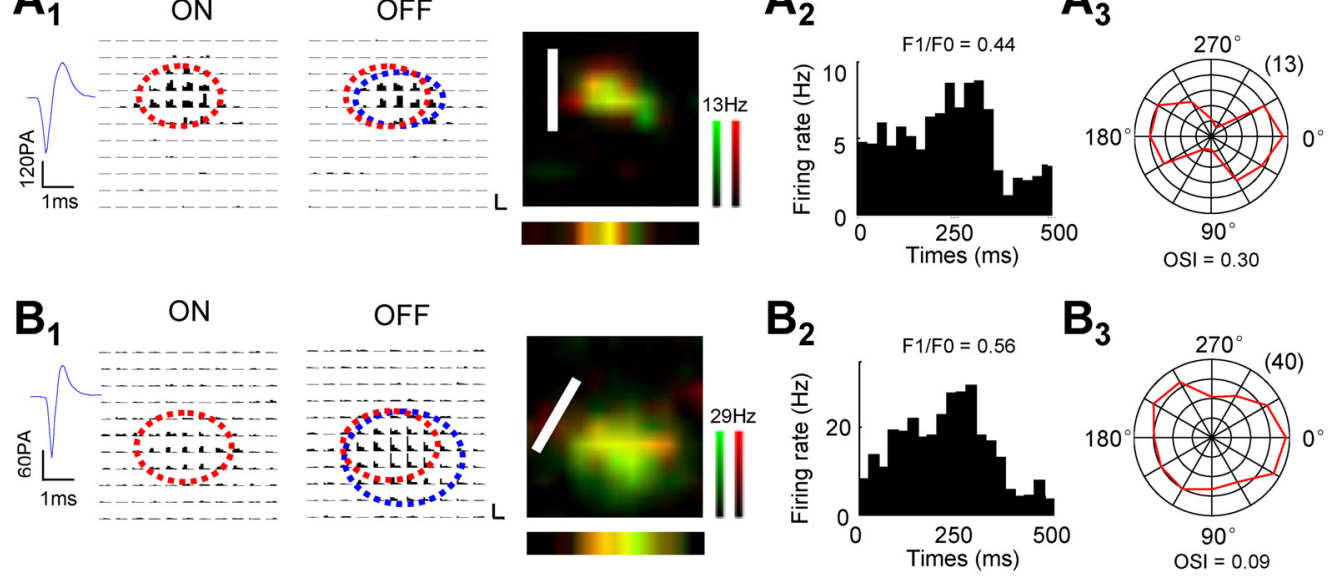

$B_{3}$
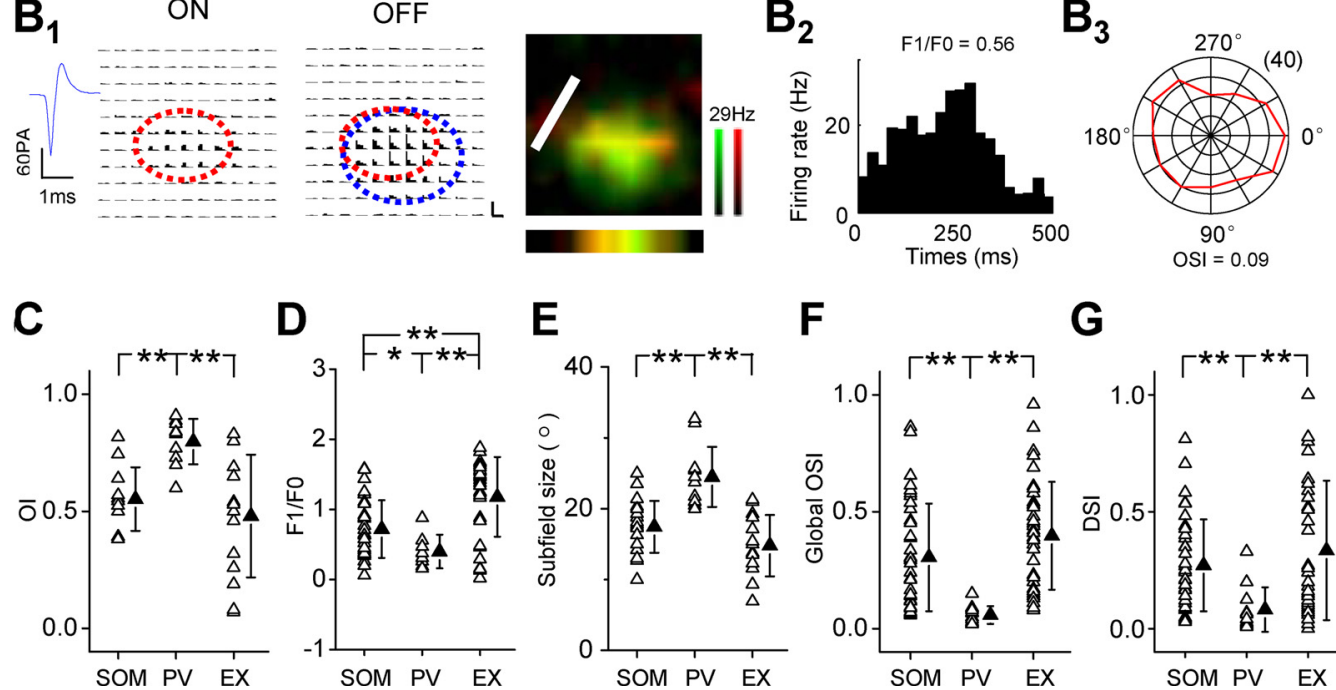

$\mathbf{F}$

G
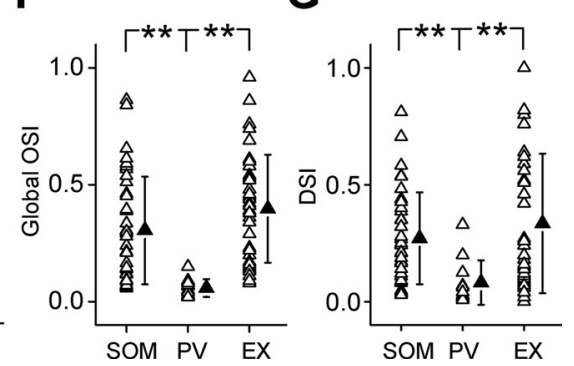

Figure 2. RF properties of SOM and PV inhibitory neurons in layer 4. $A$, An example SOM neuron. $A_{\boldsymbol{1}}$, Spatial RF. Left, Average spike shape. Middle, Array of poststimulus spike time histograms (PSTHs, bin size $=50 \mathrm{~ms}$ ) for responses to individual $0 \mathrm{n}$ or Off stimuli. Each pixel represents $5^{\circ}$ in visual space. Calibration: $17.5 \mathrm{~Hz}, 200 \mathrm{~ms}$. Right, Color map represents the superimposed spiking On (red) and Off (green) subfields. The brightness of the color represents the firing rate. The maps were smoothed by bilinear interpolation. The one-dimensional map below displays responses to flashing bars of optimal orientation (indicated by the white bar in the square map) at various locations. $A_{2}$, Cycle average of spike responses evoked by drifting sinusoidal grating at optimal spatial frequency. The modulation ratio $\mathrm{F} 1 / \mathrm{F} 0$ is indicated. $A_{3}$, Polar plot of responses to moving gratings at 12 directions. The moving direction is orthogonal to the orientation of the grating. Numbers in parentheses represent the axial scale: the average number of evoked spikes with baseline subtracted. The global orientation selectivity index (OSI) is indicated. $\boldsymbol{B}, \mathrm{An}$ example PV neuron. Data are presented in the same way as in $\boldsymbol{A}$. Calibration: $70 \mathrm{~Hz}, 200 \mathrm{~ms}$ in $\boldsymbol{B}_{1}$. C, Distribution of overlap indices (Ol) for different groups of neurons. $N=12,10$ and 14 , respectively. Solid symbol represents the mean value. Error bar, SD. ${ }^{* *} p<0.01$. D , Distribution of modulation ratios for $\mathrm{SOM}$, PV and excitatory neurons. $N=30,8$ and $22 .{ }^{*} p<0.05,{ }^{* *} p<0.01, t$ test. $\boldsymbol{E}$, Distribution of subfield sizes. The subfield size is the average of that of $\mathrm{O}$ and 0 Off subfields if both were detected. $N=20,12$, and $14 .{ }^{* *} p<0.01$. F, Distribution of 0 SI. $N=37,10$ and $34 .{ }^{* *} p<0.01$. G, Distribution of direction selectivity indices (DSI). $N=37,10$ and $34 .{ }^{* *} p<0.01$.

orientation (Fig. $2 A_{3}$, orthogonal to the direction of movement). In comparison, an example L4 PV neuron also exhibited overlapping On and Off subfields and weak modulation in its responses to sinusoidal gratings (Fig. $2 B_{1}, B_{2}$ ). However, the PV neuron showed similar levels of responses to all the orientations of the gratings (Fig. $2 B_{3}$ ), consistent with our previous results on FS neurons (Liu et al., 2009).

Spatial RFs of 37 L4 SOM neurons and 12 L4 PV neurons were examined. Among the SOM neurons, $32 \%$ of them did not show significant spiking responses to flashing stimuli, $14 \%$ had elevated spiking activity responding to some flashing stimuli but did not show a clearly identifiable spatial RF, 22\% exhibited subfields for only one contrast (monocontrast), and 32\% exhibited both On and Off subfields. In contrast to the SOM neurons, all the PV neurons exhibited clearly identifiable spatial RFs under flashing stimuli, and only $17 \%$ of them exhibited monocontrast RFs. In the inhibitory neurons that exhibited both On and Off subfields, SOM cells displayed less spatial overlap between On and Off subfields, as indicated by the significantly lower overlap indices (OI, see Material and Methods) compared with the PV neurons (Fig. $2 C)$. A majority of excitatory neurons in layer 4 have monocontrast RFs (Liu et al., 2009). For the minority of L4 excitatory neurons that displayed both On and Off subfields, their OIs exhibited a much broader variation than the inhibitory neurons (Fig. 2C). Thus, while excitatory neurons display from segregated
$(\mathrm{OI}=0)$ to completely overlapping $(\mathrm{OI}=1)$ On/Off RF structures, PV and SOM inhibitory neurons have overlapping or largely overlapping RF structures, with SOM neurons displaying less spatial overlap between On and Off subfields than PV neurons. The latter notion is further supported by the stronger modulation in the responses of SOM neurons to sinusoidal gratings than PV neurons (Fig. 2D). A majority of L4 excitatory neurons exhibited F1/F0 ratio $>1$ (Fig. $2 D$ ). This is mainly because cells with monocontrast RFs usually display strong modulation in their responses to drifting gratings (see also Liu et al., 2009). With regard to RF size, SOM neurons exhibited significantly smaller subfields than PV neurons, while similar subfield sizes as excitatory neurons (Fig. $2 E$ ).

Nearly all the SOM neurons displayed significant spiking responses to drifting gratings, indicating that SOM neurons are more sensitive to moving stimuli than to flashing stimuli. We quantified the sharpness of orientation tuning with an orientation selectivity index (OSI) derived from a global measurement (global OSI, see Materials and Methods). L4 SOM neurons exhibited a broad range of orientation tunings, from weakly tuned to strongly selective (Fig. 2 F). On the other hand, L4 PV neurons were essentially untuned to orientation, as indicated by OSI values all close to zero (Fig. 2 F). Furthermore, L4 PV neurons mostly did not show direction selectivity, as indicated by the close-to-zero direction selectivity indices (DSI, see Material and 

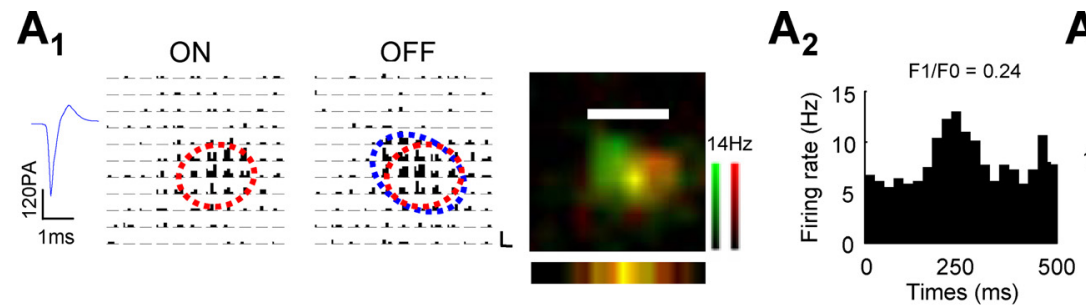

$A_{3}$
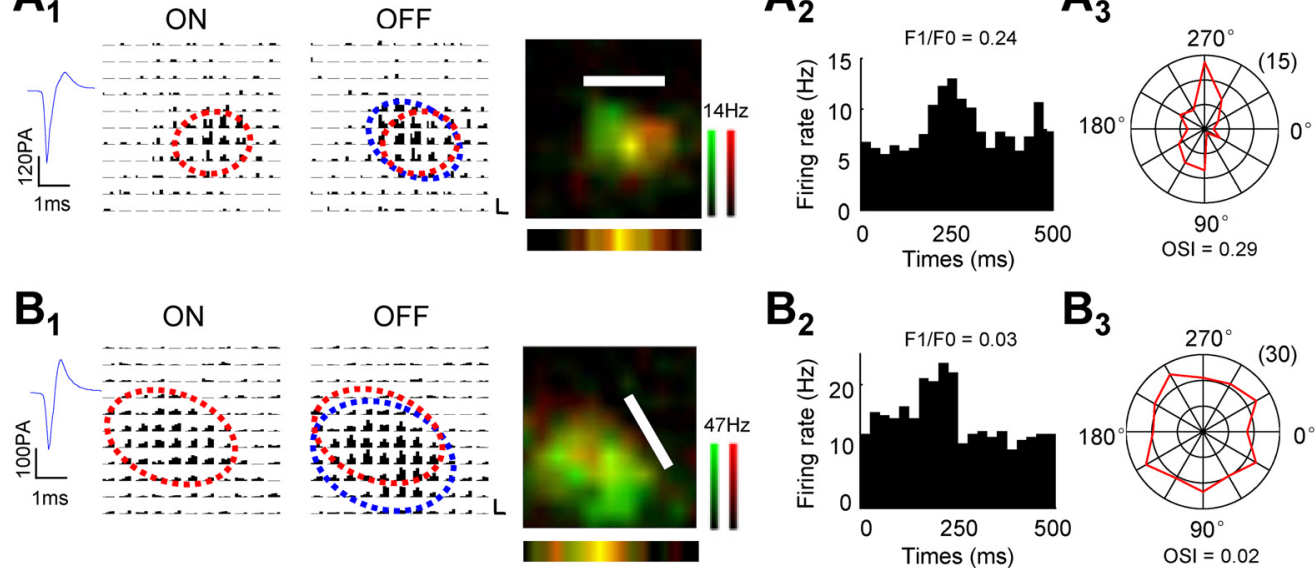

$B_{2}$

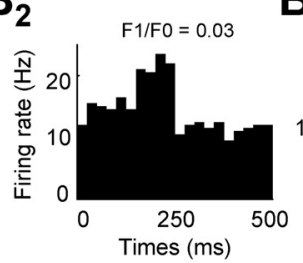

$\mathbf{B}_{3}$
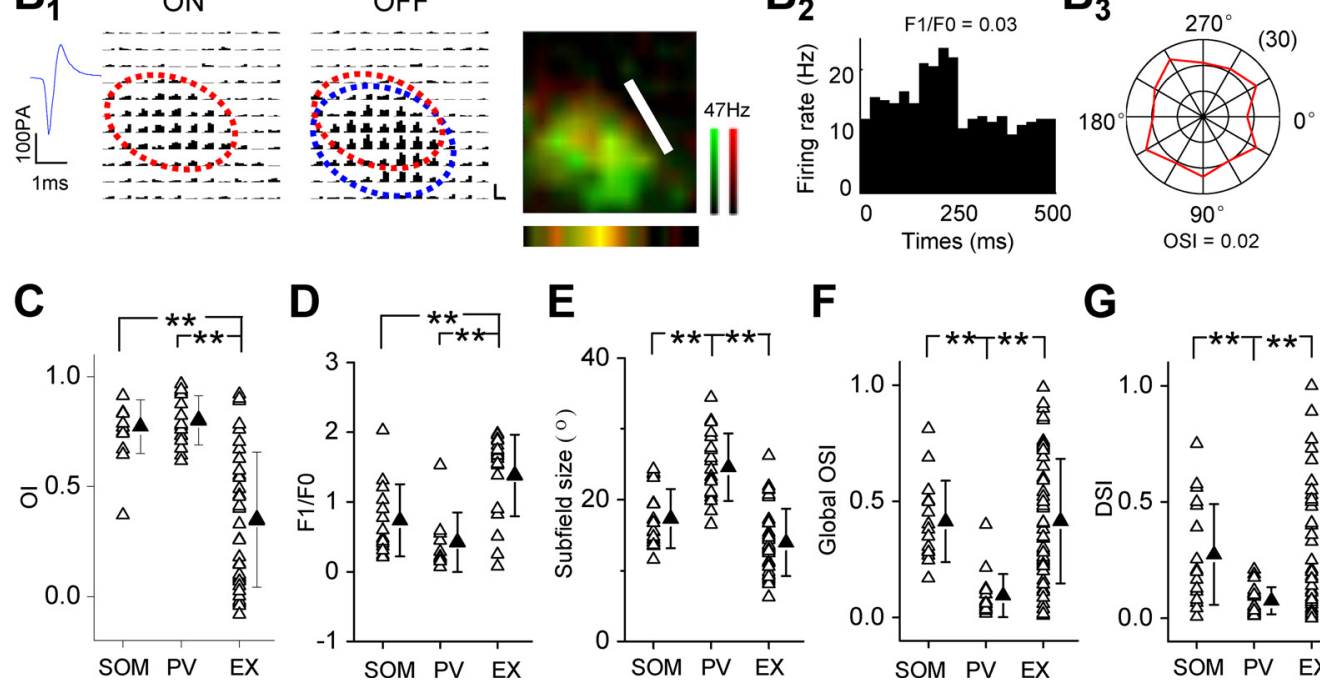

$\mathbf{F}$

G
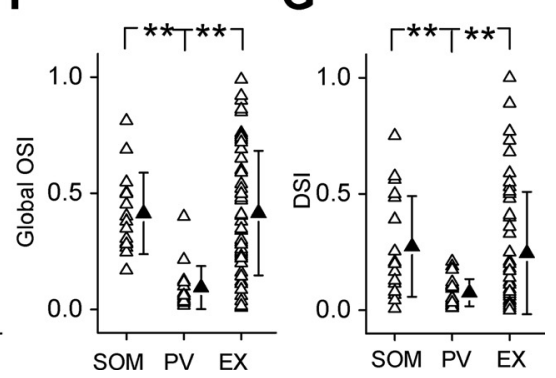

Figure 3. RF properties of SOM and PV neurons in layer 2/3. $A$, An example SOM neuron. Data are presented in the same manner as in Figure 2. $\boldsymbol{A}_{1}$, Spatial RF. Calibration: $15 \mathrm{~Hz}, 200 \mathrm{~ms}$. $\boldsymbol{A}_{2}$, Modulation in responses to sinusoidal gratings at optimal spatial frequency. $\boldsymbol{A}_{3}$, Polar plots of responses to different directions of moving gratings. $\boldsymbol{B}$, An example $\mathrm{PV}$ neuron. Calibration: $75 \mathrm{~Hz}, 200$ ms. C, Distribution of 01 for different types of neurons. $N=11,14$ and $35 .{ }^{* *} p<0.01$. D, Distribution of modulation ratio. $N=14,10$, and 31 . $E$, Distribution of subfield sizes. $N=18,20$ and 35 . $\boldsymbol{F}$, Distribution of global OSI. $N=18,17,50 .{ }^{* *} p<0.01$. G, Distribution of DSI. $N=18,17,50 .{ }^{* *} p<0.01$.

Methods), whereas SOM neurons did exhibit direction selectivity in many cells (DSI > 0.3) (Fig. 2G). Thus, in layer 4, SOM neurons have stronger selectivity to stimulus phase, orientation and direction than PV neurons.

\section{Receptive field properties of SOM and PV neurons in layer $2 / 3$}

In our previous study in layer $2 / 3$ of GAD67-GFP transgenic mice, no difference in orientation tuning was observed between FS and RS inhibitory neurons (Liu et al., 2009). However, the RS neurons in that study should be a mixture of different subtypes. Here, we further examined SOM and PV neurons in layer $2 / 3$ (L2/3). Figure 3 shows two example cells. The SOM neuron (Fig. $3 A_{1}$ ) and the PV neuron (Fig. $3 B_{1}$ ) both showed largely overlapping On and Off subfields and weak response modulation (Fig. $\left.3 A_{2}, B_{2}\right)$. However, the $\mathrm{SOM}$ neuron displayed a sharp orientation preference, while the PV neuron $\operatorname{did} \operatorname{not}\left(\right.$ Fig. $3 A_{3}, B_{3}$ ). Spatial RFs of $25 \mathrm{~L} 2 / 3 \mathrm{SOM}$ neurons and $21 \mathrm{~L} 2 / 3 \mathrm{PV}$ neurons were examined. Among the SOM neurons, $4 \%$ neurons did not show significant spiking responses to flashing stimuli, $23 \%$ did not show clearly identifiable spatial RFs, $27 \%$ exhibited monocontrast RFs and $46 \%$ exhibited both On and Off subfields. As for the PV neurons, 5\% did not show clear spatial RFs, $28 \%$ exhibited monocontrast RFs, and $67 \%$ exhibited both On and Off subfields. For cells responding to both contrasts, both PV and SOM neurons primarily showed overlapping On and Off subfields (Fig. 3C). Unlike in layer 4, there was no significant difference in the level of On/Off segregation between these two types of neurons. On the other hand, excitatory neurons exhibited a much broader variation in their RF structures, from complete On/Off segregation
$(\mathrm{OI} \leq 0)$ to complete On/Off overlap $(\mathrm{OI}=1)$ (Fig. 3C). Consistent with the structure of spatial RFs, SOM and PV neurons exhibited similarly low modulation ratios (Fig. 3D). L2/3 excitatory neurons mostly exhibited strong modulation in responses to gratings (Fig. 3D), consistent with the previous report (Niell and Stryker, 2008). It is worth noting that these cells were considered as simple cells based on modulation ratio (Niell and Stryker, 2008). However, many of these cells in fact exhibit spatially overlapping On and Off subfields characteristic of complex cells (see also Liu et al., 2009). Similarly as in layer 4, subfields of SOM neurons are significantly smaller than PV neurons (Fig. 3E).

Also similarly as in layer 4, SOM neurons exhibited significantly stronger orientation selectivity than PV neurons (Fig. $3 F$ ). In fact, SOM neurons are on average as strongly orientationtuned as excitatory neurons ( $p>0.05, t$ test, Fig. $3 F$ ), indicating that SOM neurons are capable of providing orientation-selective inhibitory inputs to principal neurons. Further more, SOM and PV neurons in layer $2 / 3$ differ in the level of direction selectivity, with SOM neurons more strongly tuned ( $p<0.05, t$ test, Fig. $3 G)$. Together, our data demonstrate that SOM neurons are most prominently different from PV neurons in their robust orientation and direction selectivity, and these functional differences are likely preserved across layers.

\section{Temporal response properties of SOM and PV neurons}

We next compared the temporal profile of evoked spiking responses between the two types of inhibitory neurons. Figure $4 \mathrm{~A}$ shows PSTHs for the responses of an example PV and SOM neuron to all the flashing stimuli during RF mapping. The firing of the PV neuron was strong and the firing rate increased rapidly 
after the stimulus onset, whereas the firing of the SOM neuron was weak with a slower rising phase. We determined the onset of evoked responses of each neuron as the time point at which firing rate exceeded 3 SDs of the baseline level (Fig. 4A, dotted lines). Comparing neurons in the same layer, we found that the onset latencies of On responses in PV neurons were significantly shorter than those in SOM neurons and excitatory neurons (L4: PV $70.8 \pm 8.5 \mathrm{~ms}$, SOM $91.8 \pm 17.8 \mathrm{~ms}, \mathrm{Ex}$ $88.9 \pm 11.0 \mathrm{~ms} ; \mathrm{L} 2 / 3: \mathrm{PV} 87.2 \pm 14.0 \mathrm{~ms}$, SOM $106.8 \pm 17.7$ ms, Ex $103.4 \pm 15.1$ ms; mean $\pm \mathrm{SD}$ ) (Fig. 4C,D). Similar observations were made for Off responses (L4: PV $72.1 \pm 10.5 \mathrm{~ms}$, SOM $98.8 \pm 22.5$ ms, Ex $89.0 \pm 10.7 \mathrm{~ms}$; L2/3: PV $95.0 \pm$ $14.1 \mathrm{~ms}$, SOM $115.7 \pm 19.7 \mathrm{~ms}$, Ex $118.1 \pm 12.1 \mathrm{~ms})$. For all the three types of neurons, response latencies in layer 4 were significantly shorter than in layer $2 / 3(p<$ 0.05 and $p<0.01$ for On and Off responses respectively, $t$ test), consistent with the direction of information flow within the cortex, i.e., from layer 4 to supragranular layers (Callaway, 1998). We next compared the onset timing of spiking responses of inhibitory neurons with that of inhibitory inputs to excitatory neurons. Inhibitory and excitatory synaptic responses to flashing stimuli were recorded under voltage-clamp recordings (Liu et al., 2010), with the cell's membrane potential clamped at $0 \mathrm{mV}$ and $-70 \mathrm{mV}$ respectively (Fig. 4B). As summarized in Figure 4,C and $D$, the onset of spiking responses of SOM neurons was significantly delayed relative to that of inhibitory input to excitatory neurons, when comparisons were made between neurons in the same layer or between L4 SOM neurons and L2/3 excitatory neurons ( $p<$ $0.05, t$ test). On the other hand, the onset of inhibitory inputs was not different from that of spiking responses of PV neurons (Fig. $4 C, D)$, suggesting that the initial inhibition onto excitatory neurons is most likely provided by PV but not SOM neurons. Furthermore, the population PSTHs for PV and SOM neurons indicate that the evoked firings of SOM cells not only started late but also peaked late compared with PV cells (Fig. 4E,F). These data provide strong in vivo evidence that $\mathrm{PV}$ neurons participate in the fast feedforward inhibition (Gabernet et al., 2005; Cruikshank et al., 2007), whereas SOM neurons provide delayed feedback inhibition (Silberberg and Markram, 2007).

\section{Discussion}

It has been shown extensively that cortical GABAergic inhibitory neurons can be categorized into distinct groups according to their expressed chemical markers. New studies have begun to reveal the potential correlation between the expression of molecular markers and the morphology and physiology of inhibitory neurons (Markram et al., 2004; Sugino et al., 2006; Dumitriu et al., 2007). Knowledge on the in vivo responses of different sub- types of inhibitory neurons is crucial for the understanding of their specific contributions to cortical processing as well as the organization of functional neural circuits. However, in almost all the previous in vivo studies in the visual cortex (Swadlow, 1988; Azouz et al., 1997; Hirsch et al., 2003; Cardin et al., 2007; Niell and Stryker, 2008; Nowak et al., 2008), the recorded presumptive inhibitory neurons were not further identified by the expression of molecular markers. Genetic labeling thus provides an invaluable tool for the targeted examination of desired inhibitory cell types.

Differential functional properties of SOM and PV neurons In this study, we applied in vivo imaging guided recordings to GFP-labeled SOM and PV neurons in specific transgenic mouse lines. We found differential response and RF properties between SOM and PV neurons. SOM neurons exhibit broader spike waveforms, much lower levels of spontaneous and evoked firing activity, smaller On/Off subfields, somewhat less spatial overlap between On and Off subfields, stronger orientation and direction selectivity as well as longer response latencies than PV neurons. 
Our results on PV neurons are in general consistent with previous studies of fast-spiking neurons (Niell and Stryker, 2008) as well as of inhibitory neurons as a population in mouse visual cortex (Sohya et al., 2007; Liu et al., 2009). Nonetheless, it should be noted that GFP expression in the mouse lines used in this study is restricted to subsets of SOM or PV expressing neurons. It remains to be further addressed whether the recordings made from these mouse lines are representative of all SOM or PV neurons. Our current study does not exclude the possibility that there are well tuned PV neurons which do not express GFP in the G42 line.

The findings on the significant levels of orientation and direction selectivity of SOM neurons are surprising and were not expected based on the previous reports, which showed that inhibitory neurons are primarily untuned or only weakly tuned to orientation (Sohya et al., 2007; Liu et al., 2009). The discrepancy between the current and previous observations may be attributed to several factors. First, as we have demonstrated in this study, the evoked spiking responses of SOM neurons are severalfold weaker than PV neurons. Since the level of $\mathrm{Ca}^{2+}$ signals highly depends on spike number and firing rate, it is possible that in the $\mathrm{Ca}^{2+}$ imaging study (Sohya et al., 2007) responses of many SOM neurons had failed to be detected due to a low signal/noise ratio. Second, it has been shown that SOM-positive cells account for almost half of non-PV inhibitory neurons (Gonchar et al., 2007). Therefore, in our earlier study (Liu et al., 2009) a significant portion of the recorded RS inhibitory neurons would presumably be SOM neurons. However, the recordings in that study likely contained sampling biases, due to a heterogeneous GFP expression level in the GAD67-GFP line and a tendency to record from brightly labeled neurons. If SOM neurons in the mouse line express GFP at low levels, the recordings could have been highly biased toward non-SOM neurons. Third, the GAD67-GFP line used in the previous studies harbors a deletion of one copy of GAD67 gene. This may affect the development of inhibitory circuits (Chattopadhyaya et al., 2007), and possibility results in reduced functional selectivity of some inhibitory neurons.

The functional differences between SOM and PV neurons may reflect different patterns of synaptic inputs they receive. Here we propose two potential mechanisms for the different orientation selectivity levels in SOM and PV neurons. In the rodent visual cortex, a systematic spatial organization of orientationtuned neurons is absent (Ohki et al., 2005). One possibility is that PV neurons unselectively receive feedforward and recurrent excitatory inputs from cells with different orientation tunings, whereas SOM neurons connect more specifically with neurons of similar orientation tunings. Another potential mechanism is suggested by the finding that SOM neurons exhibit lower levels of firing activity than PV neurons, likely due to a higher spike threshold and/or a weaker excitatory drive (Bartley et al., 2008). In any case, the selectivity of spiking responses of SOM neurons would be more greatly sharpened due to a stronger thresholding effect, even if their synaptic inputs are similarly weakly tuned as PV neurons (Priebe and Ferster, 2008; Wu et al., 2008; Liu et al., 2010).

The delayed spiking responses of SOM neurons compared with PV neurons may be attributed to two factors. First, PV neurons have been shown to receive stronger thalamocortical and intracortical excitatory inputs than SOM neurons (Gibson et al., 1999; Beierlein et al., 2003; Bartley et al., 2008; Tan et al., 2008). The EPSPs generated by these inputs also have shorter rise times in PV neurons than SOM neurons, suggesting that PV neurons can spike faster. Second, although the excitatory inputs to SOM neurons are weak, they are facilitating and can be summated during trains of high-frequency stimulation (Reyes et al., 1998; Beierlein et al., 2003; Kapfer et al., 2007; Silberberg and Markram, 2007; Bartley et al., 2008), resulting in delayed spiking of SOM neurons. Interestingly, it has been shown in vitro that under a train of high-frequency thalamocortical stimulation, spiking of PV neurons can be evoked immediately but sustains only transiently, whereas spikes of SOM neurons are activated after a significant delay but sustain more persistently (Tan et al., 2008).

\section{Functional implications on SOM and PV neurons}

The distinct RF and temporal response properties of SOM and PV neurons suggest that they may play specific roles in the functional cortical circuitry. Previous studies have shown that FS (PV) neurons are strongly and preferentially targeted by feedforward connections both from thalamus to layer 4 (Agmon and Connors, 1992; Gibson et al., 1999; Beierlein et al., 2003; Gabernet et al., 2005; Cruikshank et al., 2007) and from layer 4 to layer $2 / 3$ (Dantzker and Callaway, 2000; Xu and Callaway, 2009). FS neurons also connect strongly to their neighboring pyramidal neurons (Beierlein et al., 2003; Yoshimura and Callaway, 2005), and are thus in a good position to provide feedforward inhibition at each stage of cortical processing (Swadlow, 2003). The strong firing, fast action and perisomatic targeting of PV neurons indicates that they can tightly control the spiking of their target neurons in a timely manner (Wehr and Zador, 2003; Tan et al., 2004; Gabernet et al., 2005; Zhou et al., 2010). The largely nonselective inhibition provided by PV neurons can therefore serve as a general gain control, which may be crucial for maintaining the sharpness of orientation selectivity of excitatory neurons in the face of increasing stimulus contrast (Troyer et al., 1998; Ferster and Miller, 2000). On the other hand, SOM neurons may be more involved in providing feedback inhibition, since they do not immediately respond to ascending excitatory inputs. Interestingly, they preferentially synapse onto distal dendrites and dendritic tufts of pyramidal neurons (Kawaguchi and Kubota, 1996; Thomson and Deuchars, 1997; Somogyi et al., 1998; Di Cristo et al., 2004; Silberberg and Markram, 2007), where intracortical lateral and feedback excitatory inputs are more likely localized (Petreanu et al., 2009; Richardson et al., 2009). Late spiking and feature-selective SOM neurons are thus in a good position to locally interact with late arriving intracortical excitatory inputs and provide a more specific gating of these inputs. As the spiking responses of SOM neurons are much weaker than PV neurons, and the inhibitory synapses made by SOM neurons on pyramidal neurons are weaker than those by PV neurons (Xiang et al., 2002; Beierlein et al., 2003; Bartley et al., 2008), it seems that SOM neurons play a minor role in modulating response properties of pyramidal neurons. However, it is possible that inhibition mediated by SOM neurons can be more strongly engaged under specific stimulation paradigms that drive intensive cortical activity. To further address the functional significance of inhibitory neurons and the synaptic circuitry they participate in, it is essential to understand the nature of sensory-driven synaptic input to different subtypes of inhibitory neurons. This cannot be simply derived from the spiking response. Future in vivo intracellular experiments are required to determine the spatial and temporal patterns of excitatory and inhibitory inputs to SOM and PV neurons, respectively.

\section{References}

Agmon A, Connors BW (1992) Correlation between intrinsic firing patterns and thalamocortical synaptic responses of neurons in mouse barrel cortex. J Neurosci 12:319-329. 
Azouz R, Gray CM, Nowak LG, McCormick DA (1997) Physiological properties of inhibitory interneurons in cat striate cortex. Cereb Cortex 7:534-545.

Bartley AF, Huang ZJ, Huber KM, Gibson JR (2008) Differential activitydependent, homeostatic plasticity of two neocortical inhibitory circuits. J Neurophysiol 100:1983-1994.

Beierlein M, Gibson JR, Connors BW (2003) Two dynamically distinct inhibitory networks in layer 4 of the neocortex. J Neurophysiol 90: 2987-3000.

Callaway EM (1998) Local circuits in primary visual cortex of the macaque monkey. Annu Rev Neurosci 21:47-74.

Cardin JA, Palmer LA, Contreras D (2007) Stimulus feature selectivity in excitatory and inhibitory neurons in primary visual cortex. J Neurosci 27:10333-10344.

Chattopadhyaya B, Di Cristo G, Higashiyama H, Knott GW, Kuhlman SJ, Welker E, Huang ZJ (2004) Experience and activity-dependent maturation of perisomatic GABAergic innervation in primary visual cortex during a postnatal critical period. J Neurosci 24:9598-9611.

Chattopadhyaya B, Di Cristo G, Wu CZ, Knott G, Kuhlman S, Fu Y, Palmiter RD, Huang ZJ (2007) GAD67-mediated GABA synthesis and signaling regulate inhibitory synaptic innervation in the visual cortex. Neuron 54:889-903.

Cruikshank SJ, Lewis TJ, Connors BW (2007) Synaptic basis for intense thalamocortical activation of feedforward inhibitory cells in neocortex. Nat Neurosci 10:462-468.

Dantzker JL, Callaway EM (2000) Laminar sources of synaptic input to cortical inhibitory interneurons and pyramidal neurons. Nat Neurosci 3:701-707.

Di Cristo G, Wu C, Chattopadhyaya B, Ango F, Knott G, Welker E, Svoboda K, Huang ZJ (2004) Subcellular domain-restricted GABAergic innervation in primary visual cortex in the absence of sensory and thalamic inputs. Nat Neurosci 7:1184-1186.

Dragoi V, Sharma J, Sur M (2000) Adaptation-induced plasticity of orientation tuning in adult visual cortex. Neuron 28:287-298.

Dumitriu D, Cossart R, Huang J, Yuste R (2007) Correlation between axonal morphologies and synaptic input kinetics of interneurons from mouse visual cortex. Cereb Cortex 17:81-91.

Fairén A, DeFelipe J, Regidor J (1984) Nonpyramidal cells: general account. In: Cerebral cortex vol I (Peters A, Jones EG, eds), pp 201-253. New York: Plenum.

Felleman DJ, Van Essen DC (1991) Distributed hierarchical processing in the primate cerebral cortex. Cereb Cortex 1:1-47.

Ferster D, Miller KD (2000) Neural mechanisms of orientation selectivity in the visual cortex. Annu Rev Neurosci 23:441-471.

Freund TF, Buzsáki G (1996) Interneurons of the hippocampus. Hippocampus 6:347-470.

Gabernet L, Jadhav SP, Feldman DE, Carandini M, Scanziani M (2005) Somatosensory integration controlled by dynamic thalamocortical feedforward inhibition. Neuron 48:315-327.

Gentet LJ, Avermann M, Matyas F, Staiger JF, Petersen CC (2010) Membrane potential dynamics of GABAergic neurons in the barrel cortex of behaving mice. Neuron 65:422-435.

Gibson JR, Beierlein M, Connors BW (1999) Two networks of electrically coupled inhibitory neurons in neocortex. Nature 402:75-79.

Gonchar Y, Burkhalter A (1997) Three distinct families of GABAergic neurons in rat visual cortex. Cereb Cortex 7:347-358.

Gonchar Y, Wang Q, Burkhalter A (2007) Multiple distinct subtypes of GABAergic neurons in mouse visual cortex identified by triple immunostaining. Front Neuroanat 1:3.

Hirsch JA, Martinez LM, Pillai C, Alonso JM, Wang Q, Sommer FT (2003) Functionally distinct inhibitory neurons at the first stage of visual cortical processing. Nat Neurosci 6:1300-1308.

Kapfer C, Glickfeld LL, Atallah BV, Scanziani M (2007) Supralinear increase of recurrent inhibition during sparse activity in the somatosensory cortex. Nat Neurosci 10:743-753.

Kawaguchi Y, Kubota Y (1996) Physiological and morphological identification of somatostatin- or vasoactive intestinal polypeptide-containing cells among GABAergic cell subtypes in rat frontal cortex. J Neurosci 16:2701-2715.

Kawaguchi Y, Kubota Y (1997) GABAergic cell subtypes and their synaptic connections in rat frontal cortex. Cereb Cortex 7:476-486.
Liu BH, Li P, Li YT, Sun YJ, Yanagawa Y, Obata K, Zhang LI, Tao HW (2009) Visual receptive field structure of cortical inhibitory neurons revealed by two-photon imaging guided recording. J Neurosci 29:10520-10532.

Liu BH, Li P, Sun YJ, Li YT, Zhang LI, Tao HW (2010) Intervening inhibition underlies simple-cell receptive field structure in visual cortex. Nat Neurosci 13:89-96.

Margrie TW, Meyer AH, Caputi A, Monyer H, Hasan MT, Schaefer AT, Denk W, Brecht M (2003) Targeted whole-cell recordings in the mammalian brain in vivo. Neuron 39:911-918.

Markram H, Toledo-Rodriguez M, Wang Y, Gupta A, Silberberg G, Wu C (2004) Interneurons of the neocortical inhibitory system. Nat Rev Neurosci 5:793-807.

Mata ML, Ringach DL (2005) Spatial overlap of ON and OFF subregions and its relation to response modulation ratio in macaque primary visual cortex. J Neurophysiol 93:919-928.

Niell CM, Stryker MP (2008) Highly selective receptive fields in mouse visual cortex. J Neurosci 28:7520-7536.

Nowak LG, Sanchez-Vives MV, McCormick DA (2008) Lack of orientation and direction selectivity in a subgroup of fast-spiking inhibitory interneurons: cellular and synaptic mechanisms and comparison with other electrophysiological cell types. Cereb Cortex 18:1058-1078.

Ohki K, Chung S, Ch'ng YH, Kara P, Reid RC (2005) Functional imaging with cellular resolution reveals precise micro-architecture in visual cortex. Nature 433:597-603.

Oliva AA Jr, Jiang M, Lam T, Smith KL, Swann JW (2000) Novel hippocampal interneuronal subtypes identified using transgenic mice that express green fluorescent protein in GABAergic interneurons. J Neurosci 20:3354-3368.

Petreanu L, Mao T, Sternson SM, Svoboda K (2009) The subcellular organization of neocortical excitatory connections. Nature 457:1142-1145.

Priebe NJ, Ferster D (2008) Inhibition, spike threshold, and stimulus selectivity in primary visual cortex. Neuron 57:482-497.

Reyes A, Lujan R, Rozov A, Burnashev N, Somogyi P, Sakmann B (1998) Target-cell-specific facilitation and depression in neocortical circuits. Nat Neurosci 1:279-285.

Richardson RJ, Blundon JA, Bayazitov IT, Zakharenko SS (2009) Connectivity patterns revealed by mapping of active inputs on dendrites of thalamorecipient neurons in the auditory cortex. J Neurosci 29: 6406-6417.

Silberberg G, Markram H (2007) Disynaptic inhibition between neocortical pyramidal cells mediated by Martinotti cells. Neuron 53:735-746.

Sohya K, Kameyama K, Yanagawa Y, Obata K, Tsumoto T (2007) GABAergic neurons are less selective to stimulus orientation than excitatory neurons in layer II/III of visual cortex, as revealed by in vivo functional $\mathrm{Ca}^{2+}$ imaging in transgenic mice. J Neurosci 27:2145-2149.

Somogyi P, Tamás G, Lujan R, Buhl EH (1998) Salient features of synaptic organisation in the cerebral cortex. Brain Res Brain Res Rev 26:113-135.

Sugino K, Hempel CM, Miller MN, Hattox AM, Shapiro P, Wu C, Huang ZJ, Nelson SB (2006) Molecular taxonomy of major neuronal classes in the adult mouse forebrain. Nat Neurosci 9:99-107.

Swadlow HA (1988) Efferent neurons and suspected interneurons in binocular visual cortex of the awake rabbit: receptive fields and binocular properties. J Neurophysiol 59:1162-1187.

Swadlow HA (2003) Fast-spike interneurons and feedforward inhibition in awake sensory neocortex. Cereb Cortex 13:25-32.

Tan AY, Zhang LI, Merzenich MM, Schreiner CE (2004) Tone-evoked excitatory and inhibitory synaptic conductances of primary auditory cortex neurons. J Neurophysiol 92:630-643.

Tan Z, Hu H, Huang ZJ, Agmon A (2008) Robust but delayed thalamocortical activation of dendritic-targeting inhibitory interneurons. Proc Natl Acad Sci U S A 105:2187-2192.

Thomson AM, Deuchars J (1997) Synaptic interactions in neocortical local circuits: dual intracellular recordings in vitro. Cereb Cortex 7:510-522.

Troyer TW, Krukowski AE, Priebe NJ, Miller KD (1998) Contrastinvariant orientation tuning in cat visual cortex: thalamocortical input tuning and correlation-based intracortical connectivity. J Neurosci 18:5908-5927.

Wang Y, Toledo-Rodriguez M, Gupta A, Wu C, Silberberg G, Luo J, Markram H 
(2004) Anatomical, physiological and molecular properties of Martinotti cells in the somatosensory cortex of the juvenile rat. J Physiol 561:65-90.

Wehr M, Zador AM (2003) Balanced inhibition underlies tuning and sharpens spike timing in auditory cortex. Nature 426:442-446.

Wu GK, Arbuckle R, Liu BH, Tao HW, Zhang LI (2008) Lateral sharpening of cortical frequency tuning by approximately balanced inhibition. Neuron 58:132-143.

Xiang Z, Huguenard JR, Prince DA (2002) Synaptic inhibition of pyramidal cells evoked by different interneuronal subtypes in layer $\mathrm{v}$ of rat visual cortex. J Neurophysiol 88:740-750.

Xu X, Callaway EM (2009) Laminar specificity of functional input to distinct types of inhibitory cortical neurons. J Neurosci 29:70-85.
Xu X, Roby KD, Callaway EM (2006) Mouse cortical inhibitory neuron type that coexpresses somatostatin and calretinin. J Comp Neurol 499:144160.

Xu X, Roby KD, Callaway EM (2010) Immunochemical characterization of inhibitory mouse cortical neurons: three chemically distinct classes of inhibitory cells. J Comp Neurol 518:389-404.

Yoshimura Y, Callaway EM (2005) Fine-scale specificity of cortical networks depends on inhibitory cell type and connectivity. Nat Neurosci 8:1552-1559.

Zhou Y, Liu BH, Wu GK, Kim YJ, Xiao Z, Tao HW, Zhang LI (2010) Preceding inhibition silences layer 6 neurons in auditory cortex. Neuron 65:706-717. 